\title{
Das Sumerische ist weiterhin eine isolierte Sprache
}

Simo Parpola: Etymological Dictionary of the Sumerian Language. Part I. Lexical Evidence. Part II. Semantic Analysis and Indices. The Neo-Assyrian Corpus Project. Publications of the Foundation for the Finnish Assyriological Research. No 16/1, 16/2. 426 p. \& 436 p.

\section{Allgemeines}

Das etymologische Wörterbuch des Sumerischen von Simo Parpola (Etymological dictionary of the Sumerian language) ist ein umfangreiches, zweibändiges Werk. Es handelt sich um das erste etymologische Wörterbuch des allgemein als isolierte Sprache geltenden
Sumerischen. Die Leser der FUF könnte das Werk interessieren, weil es das Ziel verfolgt, die Verwandtschaft des Sumerischen mit den uralischen, insbesondere den ostseefinnischen Sprachen nachzuweisen. Darüber wurde auch in den finnischen Medien ausführlich berichtet (z. B. Helsingin Sanomat 16.7.).

Der erste Band enthält ein Wörterverzeichnis, das dem Verfasser zufolge insgesamt über 3000 Wortgleichsetzungen zwischen den uralischen Sprachen und dem Sumerischen auflistet. Von diesen beziehen sich 2970 auf das Ostseefinnische. Auch auf alle anderen Hauptzweige der uralischen Sprachen bezieht sich eine beträchtliche 
Zahl von Wortgleichsetzungen, u. a. auf das Saamische 1639, auf das Ungarische 1238 und sogar auf das Samojedische 891. Der zweite Band enthält denselben Wortschatz in semantische Felder gegliedert sowie einen Wortindex. Ferner enthält das Werk kurze einleitende Kapitel über die hypothetische Verwandtschaft des Sumerischen mit den finnisch-ugrischen Sprachen (im 1. Band) und über die hypothetische gemeinsame sprachliche Urheimat der finnisch-ugrischen Völker und der Sumerer (im 2. Band).

Das Sumerische ist die erste geschriebene Sprache der Welt und nimmt bei der Erforschung der sprachlichen (Vor)geschichte Eurasiens zu einem gewissen Grad eine Sonderstellung ein, denn auf sie bezieht sich die älteste sprachliche Dokumentation. Im Lauf der Forschungsgeschichte wurde versucht, das Sumerische genetisch mit vielen verschiedenen Sprachfamilien zu verbinden, etwa mit den kartwelischen, sinotibetischen, dravidischen und Munda-Sprachen, ohne allgemein anerkannten Erfolg. Auch die uralistischen Fernverwandtschaftshypothesen finden unter den Kennern der historischvergleichenden Methode in der Regel keine Unterstützung. Dies gilt u. a. für die altaische, die uraloindogermanische und die uralojukagirische Hypothese (Janhunen
1996, Koivulehto 1994, Häkkinen 2012, Aikio 2015). Da die neue Fernverwandtschaftshypothese von einem im Bereich der Sumerologie international hoch angesehenen Finnen vorgelegt wird, ist eine kritische Beurteilung der Hypothese und des sie stützenden Materials auch in einer finnougristischen wissenschaftlichen Publikation unbedingt notwendig.

Im Folgenden bemühe ich mich um ein unvoreingenommenes Urteil. Parpolas Werk verzichtet auf die bombastische Rhetorik über Wahnvorstellungen oder Verschwörungen, die für viele vom $\mathrm{Pa}$ radigma der Normalwissenschaft abweichende Publikationen charakteristisch ist. Deshalb verdient es eine ehrliche und vorurteilsfreie wissenschaftliche Beurteilung. Meine Einschätzung beruht hauptsächlich auf der uralischen Perspektive. Mein Wissen über die sumerische Sprache ist oberflächlich und beschränkt sich auf das Niveau von Handbüchern. Bei der Beurteilung der Fernverwandtschaftshypothese halte ich jedoch ihre Glaubwürdigkeit aus der Sicht der komparativen Standardmethode für zentral. Um diese zu beurteilen, muss vor allem untersucht werden, welche lautlichen und semantischen Gesetzmäßigkeiten aus dem Material hervorgehen. 


\section{Der historische Kontext}

Mit der Verwandtschaft zwischen den uralischen Sprachen und dem Sumerischen verbinden sich zahlreiche grundsätzliche Probleme der Zeit, des Raums und der Paläolinguistik. Auch der Verfasser ist sich dessen bewusst und bemüht sich, in den einleitenden Kapiteln zu Beginn des 1. und 2. Bandes auf einige von ihnen einzugehen.

Das Sumerische wurde vor rund 5000-3500 Jahren im Zweistromland im Gebiet des heutigen Irak gesprochen. Viele Forscher datieren das Ururalische auf dieselbe Periode (s. z. B. Häkkinen 2009, Kallio 2006). Das Finnische und die ostseefinnischen Sprachen, mit denen Parpola das Sumerische vor allem verbinden will, sind dagegen moderne Sprachen, und der am weitesten verbreiteten Auffassung nach spaltete sich ihre Ursprache, das Urfinnische, erst vor ca. 1500 Jahren auf, also 2000 Jahre, nachdem das Sumerische als gesprochene Sprache verschwunden war und 3500-4000 Jahre nach seiner Entstehung.

Das Ururalische und das Urfinnische unterschieden sich in Lautsystem und Wortschatz erheblich voneinander (s. z. B. Saarikivi \& Grünthal 2005). Bei einer Verwandtschaft des Sumerischen mit dem Ururalischen handelt es sich um eine schwer zu beweisende Fernverwandtschaftshypotese, bei einer Verwandtschaft mit dem Urfinnischen wiederum um die Dokumentation einer neuen ostseefinnischen Sprache. Die ostseefinnischen Sprachen stehen sich so nahe und ihre Verwandtschaft ist so offensichtlich, dass wohl niemand je die historische Verbindung z. B. zwischen dem Finnischen und dem Estnischen bewiesen hat. Wenn das Sumerische also tatsächlich eine ostseefinnische Sprache ist, müsste dies für jeden ersichtlich sein, der sich mit dem vorliegenden Wörterbuch vertraut macht.

Das Ururalische gilt in der Normalwissenschaft als Sprache der Jäger-Sammler der Steinzeit, die im Binnenland gesprochen wurde, das Urfinnische als Sprache der Eisenzeit und der Meeresküste. Die uralische sprachliche Urheimat wird in der Regel zwischen dem Wolgaknie und den südlichen Teilen Westsibiriens verortet. Das Verbreitungsgebiet des Urfinnischen wird an der Ostküste der Ostsee in der Umgebung des Finnischen Meerbusens angesiedelt, entweder auf der Südseite (Aikio 2006), in der innersten Bucht (Frog \& Saarikivi 2015) oder beidseits des Meerbusens (Itkonen 1982, Koivulehto 1983). Der Irak ist von hier ca. $4000 \mathrm{~km}$ und vom Verbreitungsgebiet des Ururalischen $2000 \mathrm{~km}$ entfernt. 
Da die Verbreitungsgebiete nahe verwandter Sprachen gewöhnlich benachbart sind, wäre die sumerisch-ostseefinnische Verbindung völlig außergewöhnlich. Zwischen Mesopotamien und der Ostsee liegen immerhin die gesamte westrussische und ukrainische Tiefebene sowie die großen Gebirge des Kaukasus und Anatoliens. Parpola präsentiert freilich im 2. Band seines Werks ein archäologisches Modell, anhand dessen sich die Verbreitungsgebiete des Ostseefinnischen und des Sumerischen historisch verbinden lassen. Das Modell beruht auf der „Kossinnaschen“ Prämisse, dass die archäologischen Kulturen der Steinzeit sprachlich recht einheitlich und identifizierbar waren. Es gibt mehrere entsprechende Hypothesen auch über die Entwicklung der uralischen Sprachfamilie, und insofern gründet sich Parpolas Darstellung auf eine Tradition. Ich habe gemeinsam mit Mika Lavento die Verwendung solcher spracharchäologischer Arealmodelle in der Finnougristik kritisiert (Lavento \& Saarikivi 2012). Unsere Kritik basiert auf der Kritik am Begriff der archäologischen Kultur und als solche auf der recht umfangreichen Literatur über die Verbindung von sprachlichem und archäologischem Material (u.a. Blench \& Spriggs 1997).
Parpola zufolge wurden die uralischen Sprachen und das Sumerische nördlich des Kaukasus gesprochen, von wo beide in ihre heutigen Gebiete wanderten. Abgesehen davon, dass die Idee im Hinblick auf die Kontaktsprachen des Ostseefinnischen fantastisch ist und auf der kritiklosen sprachlichen Identifikation der archäologischen Kulturen beruht, lässt der Verfasser den sprachlichen Kontext des Gebiets außer Acht. Wenn nämlich das Sumerische und die uralischen Sprachen Verwandte aus diesem Gebiet sind und sich nach ihnen andere Sprachen im Kaukasus verbreitet haben, müsste erörtert werden, woher diese kamen. Im Kaukasus gibt es isolierte Sprachen und kleine Sprachfamilien wie die nachisch-dagestanischen und kartwelischen Sprachen, deren große areale Diversität darauf hindeutet, dass sie bereits seit Langem in diesem Gebiet gesprochen wurden. In diesen Sprachen dürfte es auch keine Spuren von Kontakten zu den uralischen Sprachen oder dem Sumerischen geben, wohl aber zu den indogermanischen Sprachen.

\section{Aufbau und Hypothesen des Wörterbuchs}

Das Wörterbuch ist ein massives Opus von fast 1000 Seiten. Die uralisch-sumerische Hypothese wird 
also zumindest scheinbar breit untermauert. Tatsächlich ist die Zahl der ostseefinnisch-sumerischen Wortschatzparallelen größer als der auf die gemeinsame Ursprache der ostseefinnischen Sprachen, das Urfinnische, zurückgehende Wortschatz nach den üblichsten Annahmen (ca. 3000 vs. ca. 2200 Wörter - die quantitative Schätzung des urfinnischen Wortschatzes basiert auf der Auskunft von Petri Kallio). Wenn die Parallelen korrekt wären, läge also eine enge Sprachverwandtschaft vor, was die Auffassungen von der sprachlichen Vorgeschichte ganz Eurasiens auf den Kopf stellen würde. Andererseits fällt es im Licht der Analogien schwer, zu glauben, dass von einer 4000 Jahre zurückliegenden sprachlichen Verbindung 3000 Wörter in einer heute gesprochenen Sprache erhalten geblieben sein könnten. Beispielsweise umfasst nach traditioneller Auffassung der auf das Ururalische zurückgehende Wortschatz des Finnischen nur einige hundert oder sogar weniger als 200 Wörter (Sammallahti 1988, Janhunen 1981).

Der erste Band beginnt mit einer kurzen Präsentation von Parpolas Hauptthesen. In diesem $\mathrm{Zu}$ sammenhang (S. xiii-Xv) führt er Wörter an, die seiner Ansicht nach die uralisch-sumerische Verwandtschaft besonders gut erhellen. Ein großer Teil dieser Zuordnungen ist jedoch aus der Sicht der Uralistik unhaltbar. Viele umfassen junge Wörter, nicht auf das Urfinnische zurückgehende Ableitungen (fi. hälvetä 'fade away' sumerisch ha.lam-, gel.le.èm- 'perish [v]'; kiireesti 'in hurry' $\sim$ sumerisch gur.uš 'id.') oder enthalten historisch nicht zusammengehörige

uralische Wortgruppen und Lehnwörter aus bekannten Quellen (kiire 'top of a head', a Germanic borrowing kero 'hill top', a Saami borrowing Udmurt gureź 'mountain'; Finnish kähärä 'curly' Md kudrav 'id.', a borrowing from Russian $\sim \mathrm{Hu}$ göndör 'id.').

Auf Seite xiii findet sich eine Liste der Gemeinsamkeiten der uralischen Sprachen und des Sumerischen. Bei vielen handelt es sich um ausgesprochen übliche Eigenschaften in den Sprachen der Welt, beispielsweise die Verwendung von Postpositionen statt Präpositionen, die Verwendung des Ablativs bei der Komparation, die Verwendung des sein-Verbs in der Besitzkonstruktion oder die Verwendung von Partizipien. Bei anderen handelt es sich um Behauptungen, die ad hoc aufgestellt werden, ohne den Versuch, sie zu beweisen („The phonetic shapes of Sumerian words can be traced back to ProtoUralic through regular phonological changes“, „Sumerian personal, 
demonstrative, interrogative, indefinite and possessive pronouns agree with Uralic ones").

Während die traditionelle Normalwissenschaft seit fast 200 Jahren betont, dass das Ururalische eine Sprache von Jägern-Sammlern des Binnenlandes war, erklärt Parpola, in den uralischen Sprachen und im Sumerischen gebe es einen gemeinsamen Ackerbauwortschatz (2: xii), u. a. die finnischen Wörter vehnä 'Weizen' ( sumerisch ezinu 'Getreide'), ruis 'Roggen' ( ar.zig 'irgendein Getreide'), ohra 'Gerste' ( udra 'Emmer',) korjuu 'Ernte' ( 'guru, kuru'), jauho 'Mehl' ( eša 'Mehl'), multa 'Humus' ( mil, milla 'grobes Mehl') usw. Eine zweite entsprechende Behauptung besagt, dass es in den uralischen Sprachen und im Sumerischen eine gemeinsame Meeresterminologie gebe (id.), $\mathrm{zu}$ der u.a. die finnischen Wörter aapa 'Aapamoor' ( abba 'hohe See'), hyöky 'Schwall' ( ega 'Flutwelle, Strömung'), tyrsky 'Brandung' ( kur.ku 'Welle; Flut') gehören. Wer die uralische Lexikologie kennt, sieht sofort, dass sowohl der "Ackerbauwortschatz" als auch der "Meereswortschatz“ zahlreiche allgemein als entlehnt geltende Wörter, neue phonotaktische Wortstrukturtypen und junge Ableitungen enthält, für die man keine Entsprechungen im Sumerischen erwarten würde.

\section{Die Methoden}

Das vorliegende Werk ist kein etymologisches Wörterbuch im üblichen Wortsinn. Es enthält ausschließlich Wörter, für die Entsprechungen in den uralischen Sprachen vorgeschlagen werden. Ein großer Teil des sumerischen Wortschatzes wird also gar nicht behandelt, zum Beispiel Komposita oder der akkadische Lehnwortschatz, die größten Wortschatzschichten bekannter Herkunft, die Parpola zufolge insgesamt über 2500 Wörter enthalten (I: xvii, Fußnote 3).

Es handelt sich also nicht um ein Wörterbuch, sondern eher um eine Untersuchung oder ein Gedankenexperiment über die Verbindung zwischen dem Sumerischen und dem Ostseefinnischen. Aber auch als sprachgeschichtliche Untersuchung entspricht das Werk nicht der üblichen Form. Es besteht vorwiegend aus Wortvergleichen zwischen dem Sumerischen und den uralischen Sprachen, allerdings auch unter Berücksichtigung anderer Sprachen. Um die Verwandtschaft der uralischen Sprachen mit dem Sumerischen stichhaltig beweisen zu können, müsste neben Wortparallelen auch ein vollständiges System der Lautverhältnisse und Lautveränderungen vorgelegt werden, mit dem die einander zugeordneten Wörter 
regelgemäß aus einer gemeinsamen Ursprache abgeleitet werden können. Dies wird hier nicht einmal versucht. Der Verfasser räumt zwar ein, dass er einige Parallelen für wahrscheinlicher hält als andere, und im 1. Teil des Wörterbuchs hat er die Etymologien mit + oder - markiert, je nachdem, für wie wahrscheinlich er sie hält. Mit einem + versehene Etymologien bilden jedoch die überwiegende Mehrheit.

Das Werk enthält eine Lauttabelle, die dem Verfasser zufolge die Lautbeziehungen zwischen dem Sumerischen und den uralischen Sprachen darstellt (Band I, S. xxi). Fast alle Laute des Ururalischen haben in der Tabelle zahlreiche Entsprechungen in der sumerischen Graphemik, ohne dass das System der Entsprechungen kommentiert wird. Dem Laut j des Ururlaischen entsprechen Parpola zufolge in den sumerischen Texten $t, d, l, \breve{s}$ und $h$, dem Laut $k$ entsprechen $g, k, \check{s}, s$ ' und $h$ und so weiter. Da die Kontexte vieler Lautentsprechungen nicht dargelegt werden, macht die Tabelle die Verwandtschaftshypothese kaum verständlicher.

Die in der Tabelle dargestellten Lautbeziehungen stehen zudem in Widerspruch zu Parpolas Hypothese, das Sumerische sei eine ostseefinnische Sprache. Beispielsweise würde sich der ostseefinnische
Schwund der Palatalisierung im Sumerischen nicht widerspiegeln, sofern die Laute ${ }^{*} n$ und ${ }^{*} n$ ' des Ururalischen im Sumerischen durch $n$ und $n$, oder die Laute ${ }^{*} s$ ja ${ }^{*} s^{\prime}$ durch $s$ und $s^{\prime}$ vertreten sind. Auch der Laut $\check{s}$ gehört zum Lautbestand des Sumerischen und entspricht Parpola zufolge dem ururalischen Laut $\check{s}$, der nach Auffassung der Normalwissenschaft im Urfinnischen zu $h$ wurde.

Die aus der Tabelle hervorgehende Auffassung vom Lautsystem des Ururalischen weicht auch sonst von der der Normalwissenschaft ab. Parpola rekonstruiert u. a. für das „frühe Ururalische“ (die gemeinsame Ursprache des Sumerischen und der anderen uralischen Sprachen?) u. a. die Labialkonsonanten $k w$ und $\eta w$ sowie ein von den traditionellen uralischen Vokalrekonstruktionen völlig abweichendes System, in dem es keine Vokalharmonie gibt und u.a. ${ }^{*} u$ und ${ }^{\star} \ddot{u}$ auf dasselbe Phonem zurückgehen. Die vom Üblichen abweichenden Rekonstruktionen werden durch keinerlei vergleichendes Material begründet (wenn der Verfasser nicht davon ausgeht, dass der Leser nach sorgfältiger Lektüre des ganzen Wörterbuchs von ihrer Richtigkeit überzeugt ist, was zumindest bei dem unterzeichneten Leser nicht der Fall war). 


\section{Die Analyse des Materials}

Die Wortartikel bestehen fast ausschließlich aus Zuordnungen sumerischer $\mathrm{zu}$ uralischen Wörtern ohne lautliche Kommentare. Eine genauere Betrachtung der Wortartikel zeigt, dass das in den Gleichsetzungen präsentierte uralische Wortmaterial häufig aus Wörtern besteht, die nach keinem existierenden lautgeschichtlichen Modell und nach keiner diesbezüglichen Theorie zusammengehören. Wer die Methoden der Etymologie kennt, versteht, dass keinesfalls alle Gleichsetzungen zutreffen können.

Beispielsweise werden mit dem sumerischen Wort geš- 'to hear, listen, to pay attention, learn' sowohl das finnische muistaa 'remember' als auch fi. aisti 'instinct' verglichen, die jedoch ganz unterschiedlicher Herkunft sind. Zudem werden in diesem Wortartikel auch Wörter aus dem Mari und den permischen Sprachen erwähnt (mari diši- 'get used to', komi kuž 'can; be able'), die nichts mit den angeführten ostseefinnischen Wörtern oder miteinander $\mathrm{zu}$ tun haben, sowie das als saamisch bezeichnete Wort qweitse 'to notice, perceive', das mit Sicherheit nicht existiert oder nicht der saamischen Phonotaktik entspricht.

Entsprechend werden mit dem Wort sar- 'to write' sowohl fi. kirjoittaa 'schreiben' als auch fi. sorvata 'drechseln' verglichen, und mit diesen wiederum sowohl das Wort kari 'scribe' als auch das Verb sormadoms 'schreiben' der mordwinischen Sprachen, das ungarische Verb ir 'schreiben' und zahlreiche andere Wörter völlig verschiedener Herkunft. Das Wörterbuch bietet auch hier keine Hinweise darauf, inwiefern die erwähnten Wörter nach Ansicht des Verfassers zusammengehören, abgesehen von dem Zeichen +, das die Sicherheit der Etymologie markiert. Schon die Zuordnung der uralischen Wörter zueinander würde jedoch umfangreiche Kommentare erfordern - von dem sumerischen Wortvergleich ganz zu schweigen.

Die erwähnten Beispiele sind keineswegs Ausnahmen, sondern illustrieren den generellen Charakter des Wörterbuchs. Da Parpola sich bei der Rekonstruktion des Lautbestandes der Ursprachen, der uralisch-sumerischen Lautentsprechungen und des Wortschatzes der frühen Ursprachen große Freiheit gelassen hat, ist es eigentlich seltsam, dass sein Wortschatzmaterial nicht einmal diese Regelmäßigkeiten $\mathrm{zu}$ befolgen scheint. Zum Beispiel vergleicht Parpola das sumerische Wort für 'Mahlzeit', bur, mit dem finnischen Wort puuro 'Brei'. Das Homonym bur mit der 
Bedeutung 'essen' wiederum vergleicht er mit dem Verb pure- 'beißen' und das mit beiden homonyme Wort bur 'Gefäß' mit dem finnischen pursi 'Boot'. Dennoch wird das Wort buru 'Krähe' mit dem gleichbedeutenden finnischen Wort varis, das Homonym buru mit der Bedeutung 'Schild' mit dem finnischen varus 'Rüstung' und das Homonym buru 'Schar' mit dem finnischen parvi 'Schwarm'.

Der sumerischen bur-Sequenz werden also sowohl auf $v$ als auf p- anlautende Wörter mit kurzem wie mit langem Vokal zugeordnet. Dies entspricht in keiner Weise den Voraussetzungen der normalen etymologischen Forschung. Parpola erklärt zwar die Unterschiede zwischen den Entsprechungen z. B. für die sumerischen Wörter mit der Bedeutung essen, Gefäß und Mahlzeit, indem er für jedes Wort eine von den anderen abweichende Rekonstruktion anbietet, doch das erscheint recht unbegründet. Für die semantischen Veränderungen essen $\rightarrow$ Mahlzeit und essen $\rightarrow$ Gefäß finden sich viele Parallelen, und es wäre zu fragen, ob es sich nicht lediglich um Polysemie im Sumerischen handelt?

Für die Wörter parvi und varus wiederum wird ein Original vorgeschlagen, das auf den labiovelaren Klusil ${ }^{\star} k w$ anlautet. Der Verfasser versucht jedoch nicht einmal $\mathrm{zu}$ erklären, warum der labiovelare Klusil in völlig gleicher lautlicher Umgebung in einem Wort ein $p$ und im anderen ein $v$ hervorgebracht hätte. Es hat den Anschein, dass die angeführten Rekonstruktionen sich auf keinerlei systematische Auffassung von dem Lautbestand der hypothetischen Ursprache des Sumerischen und des Ostseefinnischen gründen.

Noch problematischer ist, dass die Ignorierung der bisherigen Forschung das ganze Werk durchzieht. Lexikologen im Bereich des Finnischen wissen, dass das Wort puuro auf die urfinnische Form ${ }^{\star}$ putro zurückgeht, die als baltisches Lehnwort gilt. Pursi wiederum geht auf die Form ${ }^{\star}$ purti zurück und ist eine Entlehnung aus dem Altnordischen, aus derselben Wortfamilie, der auch das englische Wort board für Schiff entstammt. Pure- geht auf das Ururalische zurück, varus ist von dem aus dem Schwedischen stammenden Wort vara abgeleitet usw. Keine dieser Etymologien wird in den Wortartikeln angeführt, es wird nicht einmal erwähnt, dass sie vorgeschlagen wurden. So geht ein normales etymologisches Wörterbuch einfach nicht vor.

Parpola schreibt zwar, dass die im Sumerischen anzutreffenden Wörter ursprüngliche uralische Wörter und keine Entlehnungen sein sollten, doch sein gewaltiges 
Material enthält reichlich Wortschatz, den alle Forscher und Wörterbücher als späte Entlehnungen aus dem Schwedischen oder Germanischen (u. a. sielu, raunio, rauta, sankari, herra, haukka, peli, kukko, kuningas, karja, laiva, lukko, muoti, nauta, naula, päärynä), dem Russischen oder Slawischen (siisti, ikkuna, kaatio, kuontalo, lusikka, piva 'Bier', pomiloi 'Gebete singen'), dem Baltischen (hauki, harja, meri) und anderen Sprachen (kehrä, jumala), als Ableitungen (hyväksyä, hyväkäs, emakko, auttava, haaveksia, innostua, jäähtyä, karhuta, kauhistua, sekoittaa, raataja, pyöristäjä, somentaa, suukottaa, syöstävä, taivaltaa), als lautlich motivierte Wörter (löperö, läpättää, läähättää, hoilata, holottaa, sirittää, sirkka, rymähtää, toitottaa) angesehen haben, zudem sogar finnische Komposita und Wortverbindungen ( $y k s i n v a l-$ tias, joukkueenjohtaja, sittapörrä, näin on, emoni kantajani, jäynän teko, näin ikään). Es entsteht der Eindruck, dass der Verfasser keine früheren etymologischen Wörterbücher oder Erstvorkommen im Finnischen oder in anderen Sprachen konsultiert hat.

Sofern der Leser die Forschung zum Wortschatz der uralischen Sprachen nicht kennt, mag er Parpolas Vergleiche mit dem Sumerischen vielleicht für begründet halten, aber wer sich auch nur mit den Grundkenntnissen vertraut macht, versteht, dass das Werk nicht der Normalwissenschaft entspricht. Um nachweisen zu können, dass zum Beispiel die „uralisch-sumerischen Ackerbau- und Meereswörter" (s. oben) korjuu, jauho, vehnä, ruis, aарa, ohra, multa, tyrsky u. a. tatsächlich auf eine alte Ursprache zurückgehen können, müsste der Verfasser erklären, was z. B. an der traditionellen Auffassung falsch ist, dass korjuu von dem Wort korjata und jauho von dem Wort jauhaa abgeleitet ist, dass das finnische aapa eine Entlehnung aus dem Saamischen ist ( saaN áhpi), und zwar von einem Wort, das seinerseits skandinavischen Ursprungs ist ( schwed. hav, s. genauer Aikio 2008), oder dass es sich bei dem finnischen multa um eine germanische Entlehnung aus dem Wort ${ }^{*}$ mulda 'Humus' handelt, das wiederum auf eine indogermanische Wurzel mit der Bedeutung zerstreuen zurückgeht $\left({ }^{*}\right.$ mel-).

Parpola begründet zu Beginn des 1. Teils seines Wörterbuchs seinen Umgang mit Lehnwörtern mit der Feststellung, dass die Entlehnungsrichtung vieler Lehnwörter unbekannt sein kann und dass ein allgemein als Lehnwort geltendes Wort zum uralischen Substrat in anderen Sprachen oder zum 
nostratischen gemeinsamen Wortschatz gehören kann (I: xxii-xxiii). In der Fußnote fügt er hinzu, dass das mordwinische gora 'Berg'

looks like a loan from Russian gorá 'mountain' (..), but it has to be taken into account that comparable words exist in many Uralic and Nostratic languages. The wide distribution of these words (..) makes a loan from Russian implausible in this case.

Aus der Sicht der normalen etymologischen Forschung ist eine solche Behauptung jedoch unsinnig: das mordwinische Wort entspricht dem russischen sowohl semantisch als auch lautlich exakt, während es keinem uralischen Erbwort in gleicher Weise entspricht. Das russische Wort hat eine präzise lautgesetzliche indogermanische Etymologie (cf. Vasmer s. v. gorá), ein uralisches Erbwort mit der Bedeutung Berg dagegen existiert nicht einmal (Saarikivi 2004). Ganz entsprechende Fälle sind kuontalo, piva, pomiloi, peli, kukko und Dutzende oder Hunderte mehr.

Wenn eine Lehnetymologie in einem völlig offensichtlichen Fall nicht gutgeheißen wird, stellt sich die Frage, ob es sich überhaupt um eine etymologische Untersuchung in der normalen Bedeutung des Wortes handelt. Die Behauptung, wonach „comparable words exist in many Uralic and Nostratic languages“, ignoriert die Grundvoraussetzung der historisch-vergleichenden normalen Sprachwissenschaft, dass nur ein Sprachvergleich, der auf der Beobachtung exakter Lautbeziehungen basiert, historisch von Bedeutung ist.

\section{Der Kernwortschatz}

Um zu klären, ob die sumerischuralische Sprachverwandtschaftshypothese irgendwie begründet sein könnte, hat der Rezensent die vorgeschlagenen sumerischen Entsprechungen des uralischen Kernwortschatzes im Licht von Parpolas Material untersucht.

Für das in fast allen uralischen Sprachen anzutreffende Wort kala wird die sumerische Entsprechung $k u$ vorgeschlagen. Für seine Reimwörter, den uralischen Verbstamm pala- 'burn', das Nomen pala 'bite' und das Nomenverbum sala 'conceal, steal' lauten die vorgeschlagenen Entsprechungen jedoch bar-, pad und hal. Obwohl es sich um Reimwörter handelt, die sich nur durch den anlautenden Konsonanten unterscheiden, sind die „Entsprechungen" völlig unterschiedlich. In dem Wort kala ist die Entsprechung des $l$ Schwund, in dem Wort palaa lautet sie $r$ und in dem Wort sala bleibt das $l$ erhalten. In dem mit dem Verb palaa homonymen Nomen pala lautet die Entsprechung wieder 
anders, nämlich $d$. Der Vokal der ersten Silbe ist aus unerfindlichen Gründen in der Entsprechung des Wortes kala ein $u$.

Wenn wir überprüfen wollten, ob der erste Konsonant des Wortes kala regelgemäß ist, könnten wir ihn mit den Entsprechungen der kivi, kuu, kaksi, kantaa und kalma vergleichen, die gin, kug, kád, gan und hilim lauten. Wir stellen fest, dass die Entsprechung des finnischen Lautes $k$ im Sumerischen offenbar sowohl $k$, als auch $g$ und $h$ sein kann und dass alle diese Entsprechungen vor einem historischen Hintervokal stehen. Andererseits ist die Entsprechung des Wortes kaikki Parpola zufolge qa.a, es kann also noch mehr Entsprechungen geben. Auch dem auf das $k$ folgenden $a$ scheinen verschiedene Vokale ohne Regelmäßigkeit zu entsprechen.

Die Entsprechung des Wortes nimi wiederum soll nam sein, was nicht völlig unmöglich klingt. Doch die Entsprechung des mit denselben Lauten beginnenden Wortes niska ist tig, die des Wortes nivoa lautet tag und als Entsprechung für das Wort nivus wird $i b$ genannt. Wie in dem vorigen Fall gibt es in ein und derselben lautlichen Umgebung mehrere Entsprechungen, zwischen denen keine Systematik zu erkennen ist. Und dasselbe gilt im Hinblick auf den gesamten alten uralischen Wortschatz. Nähdä ist Parpola zufolge igi, aber die Entsprechungen des Pronominalstamms nämä sind neund $d e$, die Entsprechung des Wortes näre ist ter und die des Wortes närhi kir. Trotz aller Anstrengungen findet selbst ein wohlwollender Leser keine Systematik.

Die historischen Lautentsprechungen sind ja eigentlich eher Entsprechungen der in den Wörtern vorkommenden Lautsequenzen als einzelner Laute. Die Entsprechung für ein und denselben Laut kann, abhängig vom Kontext durchaus unterschiedlich sein, z. B. ein Einzellaut, eine Lautverbindung, ein aus einer Lautverbindung entstandener Einzellaut usw. Dem finnischen Laut $k$ entsprechen z. B. im Ungarischen $k$ (käsi kéz), $h$ (kala hal), $g$ (tunkea $\sim d u g$ ), Schwund (jalka $\sim$ gyal-og) und so weiter. Wesentlich ist, das jede Entsprechung ihrer eigenen Kontext hat. In den Worten käsi und kala ist der auf den Laut $k$ folgende Vokal unterschiedlich (vorn gebildet vs. hinten gebildet) und die Entsprechungen sind deshalb verschieden, bei dem Wort tunkea wird die Form der Entsprechung durch den Nasal bestimmt, der dem $k$ vorangeht, und bei dem Wort jalka durch den Lateral. Doch Parpola unternimmt keinen Versuch, für die finnisch-sumerischen Wortvergleiche 
derartige Regelmäßigkeiten aufzuzeigen, und in seinem Material sind sie ebenfalls nicht zu erkennen.

\section{Zum Schluss}

Die in dem umfangreichen Werk von Simo Parpola enthaltenen uralisch-sumerischen Wortvergleiche scheinen an keine bestimmte lautgeschichtliche Theorie anzuknüpfen. Allem Anschein nach gründen sie sich hauptsächlich auf zufällige Wortassoziationen zwischen dem Sumerischen und dem heutigen Finnisch. Es sind freilich viele, und das Werk zeugt von dem enormen Eifer des Verfassers, eine beispiellose Sprachverwandtschaft zu beweisen. Bei den Vergleichen wurde jedoch leider die historisch-komparative Methode außer Acht gelassen und die Forschungsgeschichte der historischen Lexikologie der uralischen Sprachen weitgehend ignoriert.

Das Werk erinnert an den Omnikomparativismus, der vor den Junggrammatikern im 19. Jahrhundert die Hauptströmung der historischen Lexikologie war und der immer noch in gewissem Umfang begegnet (vgl. Hakola 2001), im Kreis der sog. nostratischen Hypothese teils sogar in referierten wissenschaftlichen Publikationen. Die Vertreter der historisch-vergleichenden Methode standen dem Omnikomparativismus jedoch immer kritisch gegenüber und betonten die Unabdingbarkeit regelgemäßer Lautbeziehungen für jegliche Sprachverwandtschaftshypothese.

Wenn die Lautbeziehungen des Sumerischen und der uralischen Sprachen tatsächlich regelgemäß und die uralischen Sprachen und das Sumerische verwandt sind, könnte man dies erheblich leichter beweisen. Der Verfasser könnte zum Beispiel 50 Wortpaare und eine Reihe von grammatischen Elementen suchen, in denen regelmäßige Lautbeziehungen auftreten. Über diese könnte man anstelle eines 1000 Seiten umfassenden etymologischen Wörterbuchs eine 50 Seiten lange, qualifizierte Untersuchung schreiben. Wahrscheinlich ist dies jedoch nicht möglich, denn die Sprachen sind nach Ansicht des Rezensenten nicht verwandt und ihre Lautbeziehungen sind in keiner Weise regelmäßig.

Janne Saarikivi 


\section{Literatur}

Aikio, Ante 2006: On Germanic Saami contacts and Saami prehistory. - Journal de la Société Finno-Ougrienne 91. 9-55.

- 2008: Saami loanwords in Finnish and Karelian. PhD Thesis. Oulu University.

- 2015: The Uralo-Yukaghir lexical correspondences. Genetic inheritance, language contact or chance resemblance. - Finnisch-Ugrische Forschungen 62. 7-76.

Blench, Roger \& Spriggs, Matthew 1997-2012.: Archaeology and Language. One World Archaeology Series I-III. Routledge. London \& New York.

Frog \& SAARIKIVI, JANNE 2014/2015: De situ linguarum fennicarum aetatis ferrae. - RMN Newsletter 9. 64-115.

HÄкKINEN, JАAKко 2009: Kantauralin ajoitus ja paikannus. Perustelut puntarissa. - Journal de la Société FinnoOugrienne 92. 9-56

- 2012: Early contacts between Uralic and Yukaghir. - Per Urales ad Orientem. Iter polyphonicum multilingue. Mémoires de la Société FinnoOugrienne 264. Helsinki. 91-101.

ItKonen, Terho 1983: Välikatsaus suomen kielen juuriin. - Virittäjä. 190226.

JANHUNEN, JUHA 1981: Uralilaisen kantakielen sanastosta. - Journal de la Société Finno-Ougrienne 77. 219-274.

- 1996: Fennougristiikan suhde naapuritieteisiin. - Tieteessä tapahtuu 7/1996

Kallio, Petri 2006: Suomen kantakielten absoluuttista kronologiaa. - Virittäjä 1/2006. 2-25.
Koivulehto, Jorma 1983: Seit wann leben die Urfinnen im Ostseeraum. Zur relativen und absoluten Chronologie der alten idg. Lehnwortschichten im Ostseefinnischen. - Symposium Saeculare Societatis Finno-Ugriensis. Mémoires de la Société Finno-Ougrienne 185. Helsinki. 135-157.

- 1994: Indogermanisch - Uralisch: Lehnbeziehungen oder (auch) Urverwandtschaft? - Bopp-Symposium 1992 der Humboldt-Universität zu Berlin. Akten der Konferenz vom 24.3.26.3.1992 aus Anlaß von Franz Bopps zweihundertjährigem Geburtstag am 14.9.1991. Hrsg. von Reinhard Sternemann. Winter. Heidelberg. 133-148.

LAVEnto, Mika \& SAARIKIVI, JANne 2011: Linguistics and archaeology. A critical view with an interdisciplinary approach with reference to the prehistory of Northern Scandinavia. - Charlotte Damm \& Janne Saarikivi (eds): Networks, interaction and emerging identities in Fennoscandia and beyond. Papers from the conference held in Tromsø, Norway, October 13-16, 2009. Mémoires de la Société Finno-Ougrienne 265. Helsinki. 177-217.

SAARIKIVI, JANNE \& GRÜNThal, Riho 2005: Itämerensuomalaisten kielten uralilainen tausta. - Johanna Vaattovaara et al. (eds): Muuttuva muoto. Kirjoituksia Tapani Lehtisen 6o-vuotispäivän kunniaksi. Kieli 16. Helsingin yliopiston suomen kielen laitos. Helsinki. 111-146.

Sammallahti, Pekka 1988: Historical Phonology of the Uralic Languages. - Denis Sinor (ed.): The Uralic Languages. Description, history and foreign influences. Brill. Leiden. 478-554. 